
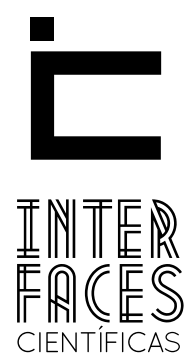

DIREITO

\title{
FIGURAS LEGAIS NA INTEGRAÇ̃̃O PRODUTIVA, ARGENTINA E BRASIL: NEGÓCIOS ASSOCIATIVOS PRA PEQUENAS E MÉDIAS EMPRESAS
}

Marta Lucila Torres Raineri ${ }^{1}$

\author{
Martín Alfredo Arjol ${ }^{2}$
}

\section{RESUMO}

O estudo analisa formas legais contratuais de cooperação empresarial da Argentina e do Brasil, para a Integração Produtiva do Mercosul, em especial pequenas e médias empresas. 0 estudo utilizou o método dedutivo, com base na descrição do âmbito do Programa de Integração Produtiva do Mercosul, colocando o caso na regulação dos diferentes regimes legais contratuais, no direito interno de cada país.

Este programa visa gerar esquemas de associação regionais, com base em projetos de negócios acordados entre empresas independentes, em um relacionamento estável ao longo do tempo. A Joint Venture é um instrumento para a associação de empresas em projetos de complementação produtiva. 0 contrato de Joint Venture poderia ser implementado em cada país, pelo acordos de complementação produtiva, Joint Venture e Consórcios de Complementação na Argentina, e do Consórcio de Empresas no Brasil. Enquanto a União Transitória Argentina e as empresas brasileiras Consórcios, têm algumas semelhanças, exigem adaptações das normas internas dos países, na condições legais semelhantes, para uma efetiva e potencial Integração Produtiva regional.

\section{PALAVRAS CHAVE}

Integração Produtiva, Negócios Associativos, Joint Ventures, Acordos de Cooperação, Consórcios. 


\section{ABSTRACT}

The study analyses contractual legal forms of business cooperation of Argentina and Brazil, for the productive integration of the Mercosur, especially small and medium-sized enterprises. Used the deductive method, on the basis of the description of the scope of productive integration program in Mercosur, placing the case in the regulation of the different contractual legal systems, in the domestic law each country. This program seeks to generate regional association schemes, based on business projects agreed on between independent enterprises, in a stable relationship over time. The Joint Venture is an instrument for the association of companies in projects of productive complementation. The contractual Joint Venture could be implemented, in every country, by Agreements of Complementation Productive, Transitory Union of Companies and Consortia of Complementation in Argentina, and the Consortium of Companies in Brazil. Although the Temporary Union of Companies Argentine and Brazilian Consortia, have some similarities, they require adjustments of the internal rules of the countries, in similar legal conditions for an effective and potential regional Productive Integration.

\section{KEYWORDS}

Productive Integration, Associative Business, Joint Venture, Cooperation Contracts, Consortia.

\section{RESUMEN}

El estudio analiza modalidades jurídicas contractuales de cooperación empresarial de Argentina y Brasil, para la Integración Productiva del Mercosur, especialmente Pequeñas y Medianas Empresas. Se utiliza el método deductivo, partiendo de la descripción de los alcances del Programa de Integración Productiva del Mercosur, situando el caso en la regulación de los diferentes regímenes jurídicos contractuales, en el derecho interno cada país. Este Programa busca generar esquemas asociativos regionales, basados en proyectos de negocios consensuados entre empresas independientes, en una relación estable en el tiempo. El Joint Venture es un instrumento para la asociación de empresas en proyectos de complementación productiva. Los Joint
Venture contractuales podrían instrumentarse, en cada país, por Acuerdos de Complementación Productiva, Unión Transitoria de Empresas y Consorcios de Complementación en Argentina, y el Consorcio de Empresas en Brasil. Si bien, la Unión Transitoria de empresas argentina y los Consorcios brasileños, poseen algunas similitudes, requieren adecuaciones de las normas internas de los países, en similares condiciones jurídicas, para una efectiva y potencial integración productiva regional.

\section{PALABRAS CLAVE}

Integración Productiva, Negocios Asociativos, Joint Ventures, Contratos de Cooperación, Consorcios. 


\section{INTRODUCCIÓN}

El proceso de integración del Mercosur se formalizó a través del Tratado de Asunción en marzo del año 1991 y estableció entre sus propósitos "el compromiso de los Estados partes de armonizar sus legislaciones en las áreas pertinentes para lograr el fortalecimiento del proceso de integración" (TRATADO DE ASUNCIÓN, 1991, n.p.).

En este sentido, el Programa de Integración Productiva del Mercosur-PIP- puesto en marcha en el año 2008, estableció una serie de acciones para cumplir con el objetivo de "[...] contribuir a fortalecer la complementariedad productiva de empresas del Mercosur, [...] a fin de profundizar el proceso de integración del bloque" (CMC, 2008, n.p.).

En un sentido amplio son mecanismos e instrumentos de políticas públicas del Mercosur, donde la cooperación y la competencia se combinan y entremezclan en la realidad del mercado, que apuntan precisamente a generar esquemas asociativos basados en proyectos de negocios consensuados entre empresas independientes, erigida por encima de las fronteras nacionales, en una relación continua o estable en el tiempo.

La armonización legislativa, específicamente contractual en la materia de contratos de cooperación empresarial, podría traer efectos beneficiosos, en generar condiciones de confianza en la cooperación e inversión de las empresas de la región.

Las integraciones económicas, (verticales u horizontales) que generan inversiones, a través de la compra de acciones o vía fusiones o absorciones, implican una unificación estratégica, y muchas veces no constituyen las respuestas adecuadas para los problemas que enfrentan las empresas, especialmente en las Pequeñas y Medianas (RODRIGUEZ, 1997, p. 5).
Las alianzas estratégicas empresariales, desde el punto de vista jurídico, pueden realizarse mediante contratos, y/o convenios de asociación o complementación, constituyendo o no, un sujeto de derecho, que puede ser distinto de sus miembros integrantes. Las formas constitutivas pueden ser, a) de base contractual, con ventajas en su flexibilidad y adaptación; o b) de base societaria, que tienen como desventaja su gran rigidez en cuanto al tipo; ya que cualquier irregularidad, traería aparejada la nulidad de las mismas y la responsabilidad personal de sus integrantes.

Según Villeneuve y Kaufman los modos en que operan la mayoría de las Alianzas estratégicas se resuelven en la forma de una pura relación contractual, y solo en ocasiones "puede ser ventajoso crear una sociedad formal o una entidad separada, por razones impositivas o de contabilidad o de limitación de responsabilidad" (MARZOTTI, 1996, p. 16). Asimismo estos autores argumentan que en la formación de una entidad separada existen más problemas creados, salvo los casos de necesidad de gerenciamiento independiente.

En este marco, el trabajo se propone examinar, modalidades jurídicas contractuales de cooperación empresarial de Argentina y Brasil, para la integración productiva del Mercosur, especialmente en las Pequeñas y Medianas Empresas-PyMes.

El artículo utiliza el método de abordaje deductivo, partiendo de la descripción de los alcances del PIP del Mercosur, analiza diferentes regímenes jurídicos y contractuales del derecho interno de los países, compara los instrumentos jurídicos y efectúa consideraciones sobre elementos convergentes y limitantes, en el ámbito de los negocios asociativos en PyMes.

\section{EL MERCOSUR Y LA INTEGRACIÓN PRODUCTIVA}

El Programa de Integración Productiva del Mercosur -PIP- es una vieja aspiración del núcleo regional y su objetivo es: 
[...] contribuir a fortalecer la complementariedad productiva de empresas del MERCOSUR, y especialmente la integración en las cadenas productivas de PYMES y de las empresas de los países de menor tamaño económico relativo, a fin de profundizar el proceso de integración del bloque, consolidando el incremento de la competitividad de los sectores productivos de los Estados Partes y otorgando herramientas adicionales que mejoren su inserción externa. (CMC, 2008, n.p.).

Las acciones de integración productiva promovidas bajo este Programa, entre otros objetivos específicos, permitiría albergar muchas formas diferentes de acuerdos de colaboración inter-empresarial, y alternativas de aplicación de contratos, como, "[...] mecanismos de desarrollo de joint ventures, clusters, entornos productivos locales, redes de proveedores y clientes, consorcios de exportación, etc." (CMC, 2008, n.p.).

El Programa enmarca las estrategias de acción a nivel horizontal y a nivel sectorial. Identifica los Joint Ventures como mecanismo de accesibilidad a los instrumentos financieros para la asociación con las empresas de los países del bloque; y en las iniciativas sectoriales de integración productiva, incluye como formas posibles de cooperación, a los Joint Ventures, en nichos específicos, y alianzas para exportación, entre otros (CMC, 2008, n.p.).

$Y$ reconoce la necesidad de articular con medidas de armonización de los sistemas jurídicas en el propósito de “[...] Analizar la creación de un marco normativo, a nivel del Mercosur, que facilite el desarrollo de empresas bi o pluri-nacionales." (CMC, 2008 , n.p.).

En este aspecto, el antecedente que apuntó a promover la integración y complementación a nivel de empresas en el Mercosur (inserta desde el proceso fundacional de Integración y Cooperación Económica entre Argentina y Brasil, 1986), se estableció en el Tratado para el Establecimiento del Estatuto de Empresas Binacionales Argentino-Brasileñas, en julio de 1990.
El Estatuto estructura un régimen jurídico especial, vigente en los estados miembros; se remite a las formas jurídicas admitidas por la legislación del país elegido para la sede social, y no "tienen personería internacional ni tampoco configuran un nuevo tipo societario de derecho interno " (BIOCCA Y RAYA DE VERA, 2008, p.164).

Este mecanismo tuvo poco impacto, dado que la forma elegida para la actuación de inversión en los países, no fue a través de las Empresas Binacionales. En la actualidad, el sistema se encuentra desactivado, por la falta de aplicación de los instrumentos de promoción fiscal, y ciertos cambios en la legislación para la actuación de sociedades extranjeras (CEPAL, 1995; BIOCCA Y RAYA DE VERA, 2008)

\section{LAS JOINT VENTURES}

La expresión “Joint Venture”, refiere a un tipo de asociación que proviene del derecho anglosajón donde se denominaban "Joint Adventures" y cuya etimología significa "Aventura Común". Es una forma de cooperación empresarial, en un contexto competitivo, que actúa como una "asociación empresarial estratégica" entre dos o más empresas nacionales y/o extranjeras, que mediante la integración, interacción y complementariedad de sus actividades y recursos, buscan alcanzar propósitos comunes.

Esta modalidad, engloba un concepto económico y viene siendo utilizada en los sistemas jurídicos latinos, con sorprendente asiduidad, para englobar institutos de muy variada índole, con importantes diferencias. Así, Cabanellas de las Cuevas y Kelly (1987) sostienen que "[...] en sentido amplio, es un instrumento de análisis económico y empresarial, empleado por los profesionales del Derecho, para hacer referencia a entes de muy variable status jurídico" (CABANELLAS DE LAS CUEVAS Y KELLY, 1987, p. 106). 
Consiste en convenios realizados por sociedades o empresarios individuales para llevar a cabo un proyecto común, que llevan implícita la asunción de un riesgo común en pos de un emprendimiento conjunto. Al respecto Le Pera se refiere como "todo acuerdo empresario para la realización de un proyecto específico, con independencia de la forma jurídica que se adopte" (LE PERA,1992, p. 83).

Esta concepción también fue utilizada, en el Tratado de Colaboración Económica firmado entre Argentina y la República de Italia (1987), para emprendimientos conjuntos de la mediana y pequeña empresa.

Es importante señalar que este tipo de contrato permite facilitar el intercambio de tecnología, como también se aplica a sectores en desarrollo, regulando la participación extranjera, en emprendimientos locales.

Si bien la mayoría de la doctrina trata de conceptualizar al “Joint Venture” desde una posición extraña al derecho anglosajón, y asimilándolo a diversas figuras de nuestro derecho, ello constituye un error, ya que el mismo es justamente en su derecho de origen, una creación jurisprudencial amparada en la ley pero utilizada con cierta ambigüedad y se aplica también este término para empresas que están interesadas en un negocio común.

Según Astolfi (1983) el término joint venture es utilizado en la práctica para mencionar acuerdos de colaboración prescindiendo de las modalidades de realización y del significado histórico y jurídico de la expresión. Con diferente enfoque Farina (1997) sostiene que los Joint Ventures no constituyen una persona jurídica porque se trata de un contrato de coordinación entre empresas y empresarios y "[...] son contratos entre sociedades que no presentan las características de estas ya que no cuentan con patrimonio propio, no son sujetos de derecho y no dan nacimiento a un ente distinto de los miembros que lo integran" (FARINA, 1997, p. 783).
Garrone y Castro Sammartino (1998) sostienen que el concepto "Joint Ventures" tiene en doctrina una gran vaguedad, sin perjuicio de lo cual enumeran una serie de notas típicas:

- Origen contractual asociativo, plurilateral que combina, estructuras u operaciones empresarias

- Realización de aportes para el negocio común, teniendo derecho a participar en las utilidades y beneficios

- Limitación del objeto del agrupamiento, en relación con el de los participantes (GARRONE Y CASTRO SANMARTINO, 1998, p. 319 e 320).

La principal clasificación distingue entre el Joint Venture contractual y el societario. El primero se formaliza y canaliza a través de un contrato celebrado entre dos o más empresas y, dada su naturaleza contractual, se excluye la creación de una sociedad o cualquier otro ente o centro de imputación normativa diferenciado. En cambio, el Joint venture societario se canaliza a través de un nuevo sujeto de derecho, particularmente una sociedad comercial, dentro de cuyo marco normativo se regirán las relaciones entre los participantes del Joint venture.

Existen dos tipos de "Joint Ventures", los que se unen contractualmente y aquellos en los que se contribuye capital. En las primeras no se necesita la formación de una tercera entidad legal en la cual los socios se obligan exclusivamente a proveer ciertos bienes o servicios u operaciones a largo plazo o a contribuir en el uso de ciertos bienes. Por lo general en la mayoría de las sociedades de coparticipación se tiende a crear una tercera entidad a los fines de cumplir con las pautas de la sociedad.

Es importante resaltar que no existen aún normas de contratación internacional que sean comunes; así Brasil no ha adherido a ningún tratado sobre derecho internacional privado, mientras que Argentina está 
enrolada en la teoría de la autonomía de la voluntad para la solución de conflictos, solución esta, negada por Brasil, salvo en lo que respecta a los contratos sometidos a arbitraje según la Ley 9.307/96 sobre Arbitraje, aunada al hecho de que Brasil haya ratificado el Protocolo de Buenos Aires sobre Jurisdicción Internacional en Materia Contractual y los Acuerdos de Arbitraje Comercial Internacional del Mercosur.

\section{FORMAS CONTRACTUALES DE COLABORACIÓN EMPRESARIA}

\subsection{ARGENTINA}

El régimen jurídico argentino se integra por diferentes figuras a través del cual las cuales pueden canalizarse estas modalidades asociativas en el campo empresarial.

La Ley de Sociedades Comerciales-LSC- No 19.550 de 1972, adoptó un sistema de tipos societarios cerrados, único medio posible dentro de la misma para estructurar relaciones de cooperación empresaria, donde la forma asociativa era sinónimo de sociedad, única herramienta idónea para estructurar las relaciones de organización empresarial.

La ley No 22.903 del año 1983, sancionó diversas reformas a la LSC, Ley No 19.550. Entre las modificaciones se incorporaron dos figuras: "las Agrupaciones de Colaboración Empresaria”-ACE- y las “Uniones Transitorias de Empresas"-UTE, en un nuevo capítulo de la ley, bajo la denominación genérica de "Los Contratos de Colaboración Empresaria"-CC, como una regulación de formas "contractuales y para societaria, que no configuran sociedades, para combinaciones mercantiles entre todo tipo de sociedades y aun de éstas con personas físicas" (Zaldivar,1984).

Posteriormente, ley No 26.005 del año 2005, se estableció la regulación del consorcio de cooperación-CC.
Richard (2013) plantea una visión más abarcadora de los negocios jurídicos para facilitar la organización y desarrollo productivo, donde la autonomía de la voluntad adquiere nuevas formas. Considera al contrato asociativo como " un vínculo de colaboración, plurilateral o de participación, con comunidad de fines, que no es sociedad" (RICHARD, 2013, p. 10)

Surgen los contratos en participación, entre los que se destacan los de colaboración o cooperación, y los de cambio con cláusulas de resultado donde hay participación en las utilidades sin configurarse sociedad.

El primero es el negocio parciario, donde se genera co-interés en el resultado del negocio, como por ejemplo en la aparcería donde no hay aportes comunes ni participación en las pérdidas y el resultado no surge de la actividad coordinada sino de la actividad de una sola de ellas. (RICHARD, 2013, p. 10).

\subsection{AGRUPACIONES DE COLABORACIÓN EMPRESARIAL-ACE}

Según los artículos No 367 a 376 de la LSC, que la facultan, poseen las siguientes características:

Su objeto consiste en facilitar- desarrollar fases de la actividad empresarial, e incrementar el resultado de esas actividades;

Se integran por sociedades argentinas, empresarios individuales o sociedades constituidas en el extranjero;

No son sociedades y no son sujetos de derecho; es una organización común;

Se inscriben en el Registro Público de Comercio.

No persiguen fines de lucro, aunque su actividad genera ventajas económicas, estas ventajas deben recaer directamente en el patrimonio de las empresas agrupadas o consorciadas. 
El contrato tienen un plazo máximo de diez años, y la modificación al contrato requiere decisión unánime;

La responsabilidad es solidaria e ilimitada con interpelación previa al administrador.

\subsection{UNIONES TRANSITORIAS DE EMPRESAS-UTE}

La Unión Transitoria de Empresas, de acuerdo a la ley 22.903, implica la reunión de las sociedades constituidas en la República, empresarios individuales domiciliados en ella y las sociedades formadas en el extranjero que hayan cumplido con el artículo No 118 LSC, mediante un contrato de unión transitoria, con el fin de desarrollo o la ejecución de una obra, servicio o suministro concretos, dentro o fuera del territorio de la República. Asimismo, la UTE podrá desarrollar o ejecutar obras y servicios complementarios y accesorios.

El contrato constitutivo bajo la forma de contrato otorgado por instrumento público o privado a inscribirse en el Registro Público de Comercio, no constituye sociedad ni es sujeto de derecho, al igual que el contrato de la ACE.

"La sociedad constituida en el extranjero se rige en cuanto a su existencia y forma por las leyes del lugar de constitución" artículo 118 LSC (lex loci celebrationis, en la cual debe comprenderse el lugar de incorporación).

El representante de la UTE, persona o personas físicas o jurídicas, tendrá los poderes de todos los miembros para ejercer los derechos y contraer las obligaciones que hiciesen al desarrollo o ejecución de la obra, servicio o suministro.

En materia de responsabilidad de las empresas participantes en la unión transitoria, no se presume la solidaridad por los actos, operaciones y obligaciones de la organización frente a terceros, salvo disposición en contrario del contrato. Los acuerdos que deban adoptar lo serán siempre por unanimidad, salvo pacto en contrario.

\subsection{CONSORCIO DE COOPERACIÓN-CC}

El consorcio de colaboración es la relación contractual que otorga a sus miembros la facultad de articular sus organizaciones empresarias, coordinando y/o relacionando diversos aspectos relativos a la fabricación, producción, administración y distribución de bienes y servicios, sin perder la individualidad de cada uno de los sujetos intervinientes (JUNYENT BAS, 2007, p. 3)

Los consorcios carecen de personalidad jurídica, no son sociedades, ni sujetos de derecho y tienen naturaleza contractual.; están destinado a personas físicas o jurídicas que se domicilien o constituyan en la Argentina, con la obligación de inscribirse en la Inspección General de Justicia o similar local o en su defecto se tomará como sociedad de hecho, entre otros (ETCHEVERRY, 2005, p. 259).

En el CC el accionar de las partes abarca una multiplicidad de operaciones y en todo el plazo de su constitución, con una funcionalidad tanto al interior como al exterior similar a la del agrupamiento de colaboración (RICHARD, 2005, p. 1).

Ninguna de las tres opciones contractuales legisladas en Argentina tiene personalidad jurídica ni constituye un nuevo sujeto de derecho. Las ACE se integran por sociedades argentinas, empresarios individuales o sociedades constituidas en el extranjero; al igual que la UTE implica la reunión de las sociedades constituidas en la República, empresarios individuales domiciliados en ella y las sociedades formadas en el extranjero; y los CC están destinado a personas físicas o jurídicas que se domicilien o constituyan en la Argentina

En la UTE el accionar de las partes se limita a operaciones concretas, determinadas y de carácter transitoria, y su plazo de ejecución no puede excederse el de la obra a ejecutar. Asimismo, su operatividad se manifiesta hacia el exterior de la obra. 
Difieren en el tema de la responsabilidad de sus miembros, que es solidaria e ilimitada para las ACE, y limitada a la proporción que fije el contrato en el caso de la UTE y los CC. El CC y la UTE no persiguen fines de lucro por sí mismas, y los beneficios económicos que genere la actividad común recaen directamente en el patrimonio de las empresas consorciadas o agrupadas. En cuanto a la responsabilidad de las partes es limitada, a la proporción que fije el contrato.

\subsection{BRASIL}

La República Federativa de Brasil, consagra la figura del consorcio en el artículo 278 de la ley $N^{\circ}$ 6404 de 1976 que dispone las Sociedades por Acciones, que establece que las compañías u otras sociedades pueden constituir contratos de consorcios para ejecutar determinados emprendimientos previstos por la ley. La legislación Brasileña no prevé personalidad jurídica para los consorcios o consorciados, solamente se obligan a las condiciones previstas en los respectivos contratos, respondiendo cada uno por sus obligaciones, sin presunción de solidaridad. Asimismo, están obligados a estar inscriptos en el Catastro Nacional de Personas Jurídicas.

La constitución de un consorcio determina que las compañías y cualquier otra sociedad, bajo el mismo control o no, pueden constituir consorcios para ejecutar un determinado emprendimiento. El consorcio funciona con un contrato entre dos o más sociedades, sean brasileñas o extranjeras, pero sin que las empresas pierdan su propia autonomía y personalidad jurídica, uniendo sus esfuerzos para obtener un objetivo común.

El consorcio tampoco tiene capacidad patrimonial, pues sus bienes pertenecen a uno o más de sus socios. Generalmente, una empresa es elegida para llevar adelante los asuntos y representar el consorcio. En la hipótesis de que haya un consorcio constituido por empresas brasileñas y extranjeras, será obligatorio que el liderazgo sea ejercido por una empresa brasileña.
El contrato de consorcio deber ser aprobado por las compañías signatarias en asamblea general, cuando se traten de sociedades anónimas. Si las compañías signatarias no son sociedades anónimas, el contrato debe ser aprobado por los respectivos órganos competentes.

Para cada sector de la economía, hay reglas distintas para la participación de los consorcios. La entidad representativa determina las normas para cada empresa que desea adquirir o participar de un emprendimiento en Brasil.

\section{LAS FIGURAS CONTRACTUALES EN LA INTEGRACIÓN PRODUCTIVA DEL MERCOSUR}

El Joint Venture es concebido dentro del Programa de Integración Productiva del Mercosur, como un instrumento para la asociación de empresas en proyectos de complementación productiva. El programa establece una serie de objetivos específicos, entre los cuales se destacan estimular, entre otros, mecanismos de desarrollo de Joint Ventures. Asimismo, establece como una medida a adoptar en materia de integración productiva sectorial la promoción de Joint Ventures en nichos específico (CMC, 2008, n.p.).

Las consecuencias beneficiosas de los Joint Ventures, en cuanto a la Integración Productiva, destacadas son: a) el acceso a nuevas tecnologías, conocimientos, equipamiento moderno y facilidades de producción, lo cual implica la modernización de la estructura productiva; b) la producción de efectos derrame (spillovers) respecto del ambiente negocial del Estado anfitrión a través de la conexión entre proveedores locales de bienes y servicios, a partir de la cual resulten procesos de innovación; c) el aumento del potencial exportador y, consecuentemente, el mejoramiento de la balanza de pagos; d) el desarrollo de nuevas y mejores capacidades y habilidades gerenciales del empresariado local necesarias para la puesta en funcionamiento 
de estrategias para la generación e incremento de competitividad, crecimiento e internacionalización, y e) el mejoramiento de las condiciones para el crecimiento de la economía local, con efectos positivos sobre el desarrollo de los recursos humanos, creación de empleo, educación, igualdad de género (UNIDO, 2008, p. 104).

Del análisis comparativo de esta figura en el ordenamiento jurídico de Argentina y Brasil, se infiere que no existe una regulación expresa como tipos asociativos, sino responden a las necesidades coyunturales económicas y desarrollo empresarial, según sea con fines de tipo contractual como de naturaleza societaria. El Joint Venture puede tomar la forma de cualquier organización societaria reconocida por las leyes argentinas y brasileñas.

Los regímenes jurídicos contractuales aplicables a los Joint Ventures se estructuran sobre la base del principio de la autonomía de la voluntad, donde las partes contratantes pueden moverse con un amplio margen de libertad para la determinación de los derechos, obligaciones y demás condiciones que regirán las relaciones recíprocas.

Pueden instrumentarse a través de un Joint Venture contractual, en la medida que califique dentro de la figura caracterizada, como las ACE, UTE o CC en Argentina, y el consorcio de empresas en Brasil, integrado supletoriamente por las normas previstas.

Las figuras existentes de corte empresarial en las normativas de Argentina y Brasil, como estados partes del Mercosur, responden independiente por cada país, en el diseño y establecimiento de sus estructuras jurídicas. Surge que existen contratos asociativos: consorcios en Brasil y tres tipos de modalidades en Argentina; Brasil, regula al Grupo de Sociedades, mientras Argentina regula a los Acuerdos de Colaboración Empresaria y a los Consorcios de Cooperación.
La Unión Transitoria de Empresas -UTE- argentina y los Consorcios brasileños, constituyen modalidades contractuales que se pueden asimilar en lo que refiere a su objeto, naturaleza, carácter, forma y régimen de responsabilidad.

La actuación de estas figuras en los países, consideradas por cada legislación como extranjeras, exigen recaudos previos de identificación, domicilio local e inscripción en el registro de comercio. Favier Dubois (1998) considera que no sería un limitante de actuación no estar inscripto en el registro de comercio, "Si su naturaleza es parciaria, [...] rigiéndose por las normas de los contratos" (FAVIER DUBOIS (h), 1998, p. 66).

Martins (2002) señala que es necesario la creación de un tipo jurídico propio para los consorcios de exportación, como el caso italiano, porque tienen mayor estabilidad, especialmente ante las singularidades de dos consorcios de exportación, que es imposible atender a través de la Ley de Sociedades por Acciones de Brasil (MARTINS, 2002, p. 15).

Arduino y Azeves (2007) argumentaron que existen propuestas tendientes a la armonización o unificación legislativa en la temática de las figuras asociativas en el ámbito jurídico del Mercosur, como: Etcheverry (2005) tomando como base el consorcio brasileño y Favier Dubois (1994) propicia una norma que disponga que, en caso de inscripción de la UTE o Consorcio en el registro del país respectivo, su naturaleza jurídica como contrato no societario, no podría ser cuestionada en ningún estado del Mercosur (ARDUINO Y AZEVES, 2007).

\section{CONCLUSIÓN}

El estudio se limita el análisis de modalidades contractuales, que constituyen una herramienta adecuada, y de utilidad para PyMes; y contribuye al conocimiento de la normativa aplicable en Argentina y Brasil. 
El Programa de Integración Productiva del Mercosur, apunta a generar esquemas asociativos regionales, basados en proyectos de negocios consensuados entre empresas independientes. Los Joint Ventures son concebidos en el Programa como un instrumento para la asociación de empresas en proyectos de complementación productiva.

Los de tipo contractual podrían instrumentarse, a nivel de cada país, en los Acuerdos de Complementación Productiva, Unión Transitoria de Empresas y Consorcios de Complementación en Argentina, y el consorcio de empresas en Brasil.

La Unión Transitoria de empresas argentina y los Consorcios brasileños, pueden tener similitudes, pero requieren adecuaciones de las normas internas de los países en similares condiciones jurídicas, a efectos de lograr una efectiva y potencial integración productiva regional.

La naturaleza esencialmente dinámica de la actividad económica requiere la necesaria adaptación del marco normativo; máxime en un proceso de Integración Regional, donde la cooperación y coordinación de las normas, de los estados integrantes, son necesarios para agilizar actividades productivas.

La armonización legislativa, específicamente en lo que respecta a los contratos de cooperación empresarial, debería tender a superar diferencias, contribuyendo a generar condiciones de confianza en la cooperación e inversión de las empresas en el avance de la Integración Productiva.

Una investigación futura sobre la efectividad de la coordinación y armonización de las normas de los contratos y/o un marco normativo, a nivel del Mercosur, en desarrollo de empresas bi o pluri-nacionales, contribuiría al conocimiento jurídico de facilitación de la organización y desarrollo productivo empresarial entre ambos países.

\section{REFERENCIAS}

ARDUINO A. H.L.; AZEVES, A. H. El marco normativo de los negocios asociativos en Mercosur. Factibilidad de determinar principios generales aplicables, como criterio unificador. El Derecho, 2 de mayo de 2007.

BIOCCA S. M.; RAYA DE VERA, E. Factores de la escasa implementación de las empresas binacionales argentino-brasileras y pautas para su reactivación. Sociedad Global, Revista de Relaciones Internacionales y Ciencias Políticas, v. 2, n. 2-3, 2008.

CABANELLAS DE LAS CUEVAS, G.; KELLY, J. A. Contratos de colaboración empresaria. Buenos Aires: Ed. Heliasta, 1987.

CEPAL. Reunión sobre Complementación Productiva, de los Países miembros del Mercosur. Santiago de Chile: Naciones Unidas,1995.

CONSEJO DEL MERCADO COMÚN (MERCOSUR). Programa de Integración Productiva del MERCOSUR. MERCOSUR/CMC/DEC. No 12/08, San Miguel de Tucumán: CMC, 2008.

ETCHEVERRY, R. A. Contratos Asociativos, negocios de colaboración y consorcios. Buenos Aires: Editorial Astrea, 2005.

FARINA, J. M. Contratos Comerciales modernos, modalidades de contratación empresaria. Buenos Aires: Editorial Astrea,1997.

FAVIER DUBOIS, E. M. La Colaboración empresaria en el Mercosur mediante los joint ventures. Aptitud general y riesgo. En: VII Congreso Argentino de Derecho Societario. III Congreso Iberoamericano de Derecho Societario y de la Empresa. La Ley, Tomo IT, Buenos Aires, 1998. 
GARRONE, J. A.; CASTRO SAMMARTINO, M. Ley de Sociedades Comerciales. Buenos Aires: Lexis Nexis - Abeledo-Perrot, 1998.

JUNYENT BAS, F. Las relaciones de organización empresaria y los llamados Consorcios de Cooperación. Academia Nacional de Derecho y Ciencias Sociales de Córdoba, 2007.

LE PERA, S. Joint venture y sociedad. Buenos Aires: Ed. Astrea, 1992.

MARTINS, G. A. Exportação de pequenas e médias empresas: problemas e sugestões para sum associativismo. Rio de Janeiro: FUNCEX, 2002.

MARZORATTI, O. J. Alianzas Estratégicas y Joint Ventures. Buenos Aires: Ed. Astrea, 1996.
RODRÍGUEZ DE RAMÍREZ, M. C. Uniones Transitorias de Empresas (U.T.E.). Buenos Aires: Ediciones Nueva Técnica S.R.L., 1997.

UNIDO- The United Nations Industrial Development Organization. Patterns of Internationalization for Developing Country Enterprises (Alliances and Joint Ventures). Viena, UNIDO, 2008.

\section{ZALDIVAR, E. Las Uniones Transitorias de Empresas.} La Ley 1984-B-919,1984.

RICHARD, E. H. Un nuevo Contrato de Colaboración Empresaria: el Consorcio de Cooperación. Academia Nacional de Derecho y Ciencias Sociales de Córdoba, 2005.

RICHARD, E. H. Las Relaciones de Organización y el Sistema del Derecho Privado patrimonial negocial (una contribución para el debate). Academia Nacional de Derecho y Ciencias Sociales de Córdoba, 2013.
1. Profesora e Investigadora, Facultad de Ciencias Económicas, Universidad Nacional de Misiones, Abogada, Especialista en Derecho de la Empresa, Posgrado en Responsabilidad Social Ambiental Empresaria, Ruta No 12, Km. 71/2, (CP 3304), Miguel Lanús, Misiones, Argentina. E-mail: raineri@ fce.unam.edu.ar, Teléfono: +54+376-154183792.

2. Docente adscripto e investigador, Facultad de Ciencias Económicas, Universidad Nacional de Misiones, Abogado, Especialista en Derecho Empresario-UNaM, Ruta No 12, Km. 71/2, (CP 3304), Miguel Lanús, Misiones, Argentina. E-mail: marjol@arnet.com.ar, Teléfono: +54+376-154606264. 